\title{
Nível crítico pelo critério da distribuição normal reduzida: uma nova proposta para interpretação de análise foliar
}

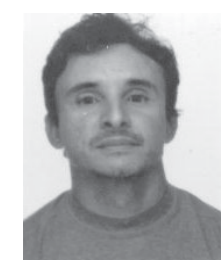

Celsemy E. Maia ${ }^{1}$, Elís R. C. de Morais ${ }^{2}$ \& Maurício de Oliveira ${ }^{3}$

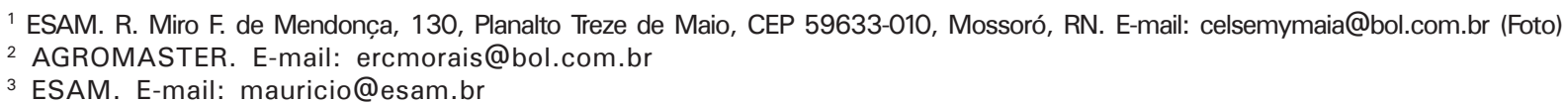

Protocolo $083-11 / 07 / 2000$

\begin{abstract}
Resumo: Objetivou-se, com este trabalho, desenvolver uma metodologia através de um embasamento estatístico, para determinação de nível crítico em tecido vegetal, oriunda de condições de campo. A obtenção do nível crítico pela distribuição contínua de probabilidade, é uma nova proposta para interpretação de análise foliar, baseada na distribuição normal reduzida. Para isto, são necessários dados de produtividade $(P)$ e de $Q$, donde $Q$ é definido como a relação entre $P$ e $\mathrm{n}_{\mathrm{i}}\left(\mathrm{Q}=\mathrm{P} / \mathrm{n}_{\mathrm{i}}\right)$, e $\mathrm{n}_{\mathrm{i}}$ é o teor do nutriente de que se deseja encontrar o nível crítico. Inicialmente encontra-se $P$ que representa $90 \%$ da máxima, pela equação $P_{(90 \%)}=1,281552 s_{1}+\bar{x}_{1}$ e para o cálculo de $Q$ que $90 \%$ do valor máximo pela equação $Q=1,281552 s_{2}+\bar{x}_{2}$ onde $\bar{x}_{1}$ e $s_{1}$ são a média aritmética e o desvio-padrão de $\mathrm{P}$ e $\overline{\mathrm{x}}_{2}$ e $\mathrm{s}_{2}$, a média e o desvio-padrão de $\mathrm{Q}$. $\mathrm{O}$ nível crítico é obtido por $\mathrm{NC}_{\mathrm{i}}=\left(1,281552 \mathrm{~s}_{1}+\overline{\mathrm{x}}_{1}\right) /\left(1,281552 \mathrm{~s}_{2}+\overline{\mathrm{x}}_{2}\right)$. O nível crítico foliar determinado pela metodologia da distribuição contínua de probabilidade permitiu calcular-se, para a cultura do café, valores dentro da faixa de referência recomendada pela literatura.
\end{abstract}

Palavras-chave: interpretação de análise foliar, nível crítico, distribuição normal

\section{Critical level through the reduced normal distribution approach: A new proposal for interpretation of foliar analysis}

\begin{abstract}
This study develops a methodology through a statistical method, for the determination of critical level in foliar analysis. The obtaining of the critical level with the continuous distribution of probability is a new proposal for foliar analysis interpretation, based on the reduced normal distribution. For this purpose it is necessary to have data of productivity $(P)$ and of $Q$, defined as the relationship between $P$ and $n_{i}\left(Q=P / n_{i}\right)$, where $n_{i}$ is the content of the nutrient for which critical level is to be found. Initially $P_{r}$ which represents $90 \%$ of the maxim, is calculated with the equation $\mathrm{P}(90 \%)=1.281552 \mathrm{~s}_{1}+\mathrm{x}_{1}$ and for the calculation of $\mathrm{Q} 90 \%$ of the maximum value with the equation $\mathrm{Q}=1.281552 \mathrm{~s}_{2}+\mathrm{x}_{2}$ where $\mathrm{x}_{1}$ and $\mathrm{s}_{1}$ are the arithmetic average and the standard deviation of $\mathrm{P}$ and $\mathrm{x}_{2}$ and $\mathrm{s}_{2}$ the average and the standard deviation of $\mathrm{Q}$. The critical level is obtained by $\mathrm{NCi}=\left(1.281552 \mathrm{~s}_{1}+\mathrm{x}_{1}\right) /\left(1.281552 \mathrm{~s}_{2}+\mathrm{x}_{2}\right)$. The critical level methodology of the continuous distribution of probability allowed the calculation, for the coffee crop, of critical values within range of those recommended in the literature.
\end{abstract}

Key words: foliar analysis interpretation, critical level, normal distribution

\section{INTRODUÇÃO}

Para os pesquisadores em fertilidade do solo e nutrição de plantas, a avaliação do estado nutricional das culturas tem sido um desafio, principalmente em locais onde ocorrem limitações na produtividade pelo desequilíbrio nutricional. A contribuição da diagnose foliar levou pesquisadores a desenvolverem diversas metodologias de interpretação de resultados de análise foliar, dentre as quais se encontra o nível crítico, definido como sendo a concentração do nutriente no tecido vegetal, acima do qual pequenos ou nenhum aumento na produção é esperado (Escano et al., 1981) ou a concentração que separa populações de baixa e alta probabilidade de resposta à adição do nutriente. Baseia-se na premissa de que existe uma relação direta entre os teores dos nutrientes no tecido das plantas e a sua produção, visto que, dentro de alguns limites, existe uma relação direta entre o nutriente fornecido pelo solo ou pelo adubo e a produção; também dentro de limites, há uma relação direta entre o nutriente fornecido pelo solo ou pelo adubo e a sua concentração nas folhas; acontece uma relação direta entre a 
concentração do nutriente nas folhas e a produção (Orlando Filho \& Zambello, 1983).

A forma tradicional de se obter níveis críticos passa por ensaio de calibração da produtividade vegetal em função do teor de um nutriente na planta. Segundo Walworth \& Sumner (1988) muitos pesquisadores conduzem experimentos variando a dose do nutriente no solo, obtendo a produtividade e a concentração do nutriente no tecido vegetal. Essas concentrações são ajustadas em função das produtividades, através de modelos matemáticos, como Mitscherlich, quadrática, exponencial etc. Em muitos casos, o nível crítico corresponde à concentração do nutriente, referente a 90\% da produtividade máxima. Para a CFSEMG (1989) a determinação dos "níveis críticos" para os diversos nutrientes em relação às diversas culturas, é uma das fases da diagnose foliar que demanda grande esforço por parte da pesquisa.

Existem métodos de interpretação foliar que não necessitam de ensaio de calibração no campo. Wadt (1996) desenvolveu o Método da Chance Matemática que apresenta como vantagem, em relação ao método tradicional para determinação do nível crítico, a origem dos dados e possibilita a utilização de dados oriundos de condições de campo, e não apenas de ensaio controlado. Portanto, as exigências do controle experimental podem ser ignoradas, contanto que haja uma quantidade relativamente grande de informações sobre o fator a ser analisado.

Objetivou-se, com este trabalho, desenvolver uma metodologia com embasamento estatístico, para determinação de nível crítico em tecido vegetal, oriunda de condições de campo.

\section{METODOLOGIA}

A distribuição normal ou de Gauss-Laplace, é uma das mais importantes distribuições teóricas contínuas de probabilidade, aplicada em inúmeros fenômenos e constantemente utilizada para o desenvolvimento teórico da inferência estatística. A função que descreve a forma da curva é a Eq. 1, onde $\mu$ é a média e $\sigma$ o desvio-padrão é denotada por $\mathrm{N}(\mu, \sigma)$.

$$
f(x)=\frac{1}{\sigma \sqrt{2 \pi}} e^{-\frac{1}{2}\left(\frac{x-\mu}{\sigma}\right)^{2}},-\infty<x<+\infty
$$

Para o cálculo da probabilidade, surgem dois grandes problemas: primeiro, para a integração de $\mathrm{f}(\mathrm{x})$, pois para o cálculo é necessário o desenvolvimento em séries; segundo, seria a elaboração de uma tabela de probabilidade, pois $\mathrm{f}(\mathrm{x})$ depende de dois parâmetros, fato este que acarretaria um grande trabalho para se tabelar essas probabilidades, considerando-se as várias combinações de valores de $\mu$ e $\sigma$. Os problemas foram resolvidos por meio de uma mudança de variável obtendo-se, assim, a distribuição normal padronizada ou reduzida (Fonseca \& Martins, 1990). A variável reduzida (Z) é dada pela Eq. 2, em que X é uma variável normal de média $\mu$ e desvio-padrão $\sigma$. A distribuição normal reduzida possui média zero e variância um - $\mathrm{N}(0,1)$.

$$
\mathrm{Z}=\frac{\mathrm{X}-\mu}{\sigma}
$$

Para se calcular o nível crítico pela distribuição contínua de probabilidade, as variáveis utilizadas na metodologia são:

$\mathrm{P} \quad$ - produtividade $\left(\mathrm{Mg} \mathrm{ha}^{-1}\right.$, sacos ha ${ }^{-1}$, caixas ha-1 etc.),

$\mathrm{n}_{\mathrm{i}} \quad$ - teor no nutriente i na folha (dag $\mathrm{kg}^{-1}$ ou $\mathrm{mg} \mathrm{kg}^{-1}$ ),

$\mathrm{Q}-\mathrm{P} / \mathrm{n}_{\mathrm{i}}$,

$\mathrm{P}_{(90)}$ - valor crítico da produtividade acima da qual se espera sob a hipótese de normalidade, que sejam observados apenas $10 \%$ das produtividades,

$\mathrm{Q}_{(90)}$ - valor crítico do quociente $\mathrm{Q}$, acima do qual se espera sob hipótese de normalidade, que sejam observados apenas 10\% dos valores de Q.

Sob a hipótese de que $\mathrm{P}$ segue a distribuição normal com média $\mu_{\mathrm{p}}$ e variância $\sigma_{\mathrm{p}}{ }^{2}$, denotada por $\mathrm{P} \sim \mathrm{N}\left(\mu_{\mathrm{p}}, \sigma_{\mathrm{p}}{ }^{2}\right)$, segue que a variável $\mathrm{Z}$ possui distribuição normal com média zero e variância um (Eq. 3).

$$
Z=\frac{P-\mu_{p}}{\sigma_{p}} \sim N(0,1)
$$

Por outro lado, o valor $\mathrm{P}_{(90)}$ pode ser determinado por meio do seguinte procedimento:

$$
\begin{aligned}
\mathrm{P}(\mathrm{P} & \leq \mathrm{P}(90))=\mathrm{P}\left(\frac{\mathrm{P}-\mu_{\mathrm{p}}}{\sigma_{\mathrm{p}}} \leq \frac{\mathrm{P}_{(90)}-\mu_{\mathrm{p}}}{\sigma_{\mathrm{p}}}\right) \\
& =\mathrm{P}\left(\mathrm{Z} \leq \frac{\mathrm{P}(90)-\mu_{\mathrm{p}}}{\sigma_{\mathrm{p}}}\right)=0,90
\end{aligned}
$$

Da tabela de distribuição normal padrão, tem-se que:

$$
\mathrm{P}(\mathrm{Z} \leq 1,281552)=0,90
$$

Comparando-se a Eq. (4) com (5), concluímos que:

$$
\frac{\mathrm{P}(90)-\mu_{\mathrm{p}}}{\sigma_{\mathrm{p}}}=1,281552 \Rightarrow \mathrm{P}(90)=1,281552 \sigma_{\mathrm{p}}+\mu_{\mathrm{p}}
$$

De modo análogo, determina-se $\mathrm{Q}_{(90)}$ pela Eq. 7.

$$
\mathrm{Q}(90)=1,281552 \sigma_{\mathrm{q}}+\mu_{\mathrm{q}}
$$

Supondo-se que, até certo ponto, a produtividade P é proporcional ao teor de nutriente i na folha, então o quociente Q e o nível crítico $n_{i}$ são definidos por:

$$
\mathrm{Q}=\frac{\mathrm{P}}{\mathrm{n}_{\mathrm{i}}} \Rightarrow \mathrm{n}_{\mathrm{i}}=\frac{\mathrm{P}}{\mathrm{Q}}
$$

Constata-se que o nível crítico do nutriente i, que determina o valor da produtividade que representa $90 \%$ do seu valor máximo, é obtido pela Eq. 9 .

$$
\mathrm{n}_{\mathrm{i}}=\frac{\mathrm{P}(90)}{\mathrm{Q}(90)}=\frac{1,281552 \sigma_{\mathrm{p}}+\mu_{\mathrm{p}}}{1,281552 \sigma_{\mathrm{q}}+\mu_{\mathrm{q}}}
$$


Tabela 1. Valores de média, desvio-padrão e níveis críticos para café conilon, em lavouras do Estado do Espírito Santo, obtidos pelo método da distribuição contínua de probabilidade

\begin{tabular}{|c|c|c|c|c|c|}
\hline \multirow{2}{*}{$\begin{array}{l}\text { Nutriente }^{1 /} \\
\text { Produtividade }\end{array}$} & \multirow[b]{2}{*}{ Média } & \multirow[b]{2}{*}{ Desvio-Padrão } & \multirow{2}{*}{$\begin{array}{l}\text { Nível Crítico } \\
\text { Calculado }^{3}\end{array}$} & \multicolumn{2}{|c|}{ Nível Crítico da Literatura } \\
\hline & & & & $\begin{array}{l}\text { Trani et al. (1983) } \\
\text { e Raij (1991) }\end{array}$ & Jones Jr. et al (1991) \\
\hline Produtividade $^{2}$ & 2,683 & 0,709 & - & - & \\
\hline $\mathrm{N}$ & 1,641 & 0,727 & 2,77 & $2,80-3,00$ & $2,50-3,00$ \\
\hline $\mathrm{P}$ & 4,830 & 0,720 & 0,12 & $0,12-0,15$ & $0,15-0,35$ \\
\hline $\mathrm{K}$ & 1,999 & 0,733 & 1,92 & $1,80-2,00$ & $2,00-3,00$ \\
\hline $\mathrm{Ca}^{(*)}$ & 3,578 & 1,227 & 1,37 & $1,00-1,50$ & $0,8-1,60$ \\
\hline $\mathrm{Mg}$ & 3,831 & 0,743 & 0,30 & $0,35-0,40$ & $0,30-0,50$ \\
\hline $\mathrm{S}$ & 4,118 & 0,709 & 0,24 & $0,20-0,24$ & $0,25-0,50$ \\
\hline $\mathrm{Fe}$ & $-1,955$ & 0,758 & 97,01 & $70-100$ & $90-300$ \\
\hline $\mathrm{Zn}$ & 0,327 & 0,726 & 10,32 & $10-15$ & $15-200$ \\
\hline $\mathrm{Mn}$ & $-1,461$ & 0,930 & 47,50 & $50-100$ & $50-300$ \\
\hline $\mathrm{B}$ & $-1,147$ & 0,806 & 40,66 & $40-60$ & $25-75$ \\
\hline $\mathrm{Cu}$ & 0,360 & 1,014 & 6,89 & $6-10$ & $10-50$ \\
\hline
\end{tabular}

Produtividade para $90 \%$ da máxima

36,28 sacas $^{-1} a^{-1}$

${ }^{1}$ Os dados das médias e desvios-padrão para cada nutriente e produtividade/n foram transformados por ln, com exceção da relação $P / \mathrm{Ca}^{(*)}$, transformada usando-se a raiz quadrada

${ }^{2}$ A média e o desvio-padrão da produtividade (sacas ha $\left.{ }^{-1}\right)$ são para transformação $\ln$ (produtividade)

${ }^{3}$ Os valores dos níveis críticos de $\mathrm{N}, \mathrm{P}, \mathrm{K}, \mathrm{Ca}, \mathrm{Mg}$ e S estão em dag kg-1 e Fe, Zn, Mn, B e Cu em mg kg-

Como, em geral, não se conhecem $\mu_{\mathrm{p}}, \mu_{\mathrm{q}}, \sigma_{\mathrm{p}}$ e $\sigma_{\mathrm{q}}$, tomam-se as suas estimativas $\overline{\mathrm{x}}_{\mathrm{p}}, \overline{\mathrm{x}}_{\mathrm{q}}, \mathrm{s}_{\mathrm{p}}$ e $\mathrm{s}_{\mathrm{q}}$, respectivamente, obtidas de amostras suficientemente grandes; assim, uma estimativa do nível crítico é dada por:

$$
\hat{\mathrm{n}}_{\mathrm{i}}=\frac{1,281552 \mathrm{sp}+\overline{\mathrm{x}}_{\mathrm{p}}}{1,281552 \mathrm{sq}+\overline{\mathrm{x}}_{\mathrm{q}}}
$$

ou pelas Eqs. 11 ou 12, para dados transformados em ln ou raiz quadrática, respectivamente.

$$
\begin{gathered}
\hat{\mathrm{n}}_{\mathrm{i}}=\frac{\operatorname{Exp}(1,281552 \mathrm{sp}+\overline{\mathrm{x}} \mathrm{p})}{\operatorname{Exp}(1,281552 \mathrm{sq}+\overline{\mathrm{x}} \mathrm{q})} \\
\hat{\mathrm{n}}_{\mathrm{i}}=\frac{(1,281552 \mathrm{sp}+\overline{\mathrm{x}} \mathrm{p})^{2}}{\left(1,281552 \mathrm{sq}+\overline{\mathrm{x}}_{\mathrm{q}}\right)^{2}}
\end{gathered}
$$

A pressuposição básica para se encontrar o nível crítico pela distribuição contínua de probabilidade, é a de que os dados de P e Q sigam distribuição normal. Para isto, pode-se testar a normalidade das variáveis citadas por meio de testes como Qui-quadrado (Snedecor \& Cochran, 1973) ou Lilliefors (Conover, 1971).

\section{RESULTADOS E DISCUSSÃO}

Os valores das análises foliares para exemplificar o método, foram retirados do trabalho de Leite (1993). O banco de dados de café foi composto por 157 análises foliares do Estado do Espírito Santo, para 11 nutrientes, com as respectivas produtividades de cada plantio.

Após aplicar-se o teste do qui-quadrado, observou-se que a produtividade não apresentou normalidade e os dados foram transformados por $\ln (\mathrm{P})$. Todas as relações $\mathrm{P} / \mathrm{n}_{\mathrm{i}}$ foram também transformadas por $\mathrm{ln}$, com exceção da relação $\mathrm{P} / \mathrm{Ca}$, que foi transformada usando-se a raiz quadrada. O nível crítico para cálcio foi encontrado pela Eq. 13 e, para os demais nutrientes, pela Eq. 14.

$$
\begin{gathered}
\hat{\mathrm{n}}_{\mathrm{i}}=\frac{\mathrm{e}^{(1,281552 \mathrm{sp}+\mathrm{xp})}}{\left(1,281552 \mathrm{sq}+\overline{\mathrm{x}}_{\mathrm{q}}\right)^{2}} \\
\hat{\mathrm{n}}_{\mathrm{i}}=\frac{\mathrm{e}^{(1,281552 \mathrm{sp}+\mathrm{xp})}}{\mathrm{e}^{(1,281552 \mathrm{sq}+\mathrm{xq})}}
\end{gathered}
$$

Os valores de média e desvio-padrão de cada nutriente e seu respectivo nível crítico, estão na Tabela 1, e mostram que não houve discrepância dos citados por Trani et al. (1986) e van Raij (1991) indicando boa estimação. Os níveis críticos para nitrogênio e magnésio ficaram abaixo dos valores recomendados por Trani et al. (1986) e van Raij (1991) porém dentro do intervalo sugerido por Jones Jr. et al. (1991).

Além dos níveis críticos estarem de acordo com a literatura, o método apresenta a vantagem de não se necessitar de ensaio de calibração para determinação do nível crítico para as culturas. Possuindo um banco de dados com um número de análise foliar que represente determinada área ou região e que, na mesma amostra foliar, sejam determinados vários nutrientes, o método permite encontrar-se os níveis críticos para todos os elementos analisados e a faixa de suficiência bastando para isto alterar-se a constante relativa à produtividade de $90 \%$ da máxima, para um valor desejado.

\section{CONCLUSÃO}

O nível crítico foliar determinado pela metodologia da distribuição contínua de probabilidade permitiu calcular-se, para a cultura do café, valores dentro da faixa de referência recomendada pela literatura.

\section{LITERATURA CITADA}

CFSEMG - Comissão de Fertilidade do Solo do Estado de Minas Gerais. Recomendação para o uso de corretivos e fertilizantes em Minas Gerais. $4^{\mathrm{a}}$ aproximação. Lavras, 1989. 159p. 
Conover, N.J. Practical non parametric statistics. New York: John Wiley, 1971.462p.

Escano, C.R.; Jones, C.A.; Uehara, G. Nutrient diagnosis in corn grown on Hidric Dystrandepts: I. Optimum tissue nutrient concentrations. Soil Science Society America Journal, Madison, v.45, p.1135-1143, 1981.

Fonseca, J.S.; Martins, G.A. Curso de estatística. 3.ed, São Paulo: Atlas, 1990. 286p.

Jones Jr., J.B.; Wolf, B.; Mills, H.A. Plant analysis handbook - a practical sampling, preparation, analysis and interpretation guide. Athens: Micro-macro Publishing, 1991, 213p.

Leite, R. de A. Avaliação do estado nutricional do cafeeiro conilon no Estado do Espírito Santo, utilizando-se diferentes métodos de interpretação de análise foliar. Viçosa:UFV, 1993. 87p. Tese Doutorado
Orlando Filho, J.; Zambello Jr., E. Diagnose foliar. In: Orlando Filho, J. (ed) Nutrição e adubação da cana-de-açúcar no Brasil. Piracicaba: IAA/Planalsucar, 1983, p.125-152.

Snedecor, G.W.; Cochran, W.G. Statistical methods. 6.ed. Iowa: University Press, 1973. 593p.

Trani, P.E.; Hirose, R.; Bataglia, O.C. Análise foliar: Amostragem e interpretação. Campinas: Fundação Cargill, 1983, 18p.

van Raij, B. Fertilidade do solo e adubação. Piracicaba: CERES/ POTAFOS, 1991.343p.

Wadt, P.G.S. Os métodos da chance matemática e do sistema integrado de diagnose e recomendação (DRIS) na avaliação nutricional de plantas de eucalipto. Viçosa: UFV, 1996, 123p. Tese Doutorado

Walworth, J.L.; Sumner, M.E. Foliar diagnosis: A review. Advances in Plant Nutrition, New York, v.3, p.193-241. 1988. 